\title{
Adaptation of Temporal Control to Unpredictable Mid-Session Changes in a Rapid-Acquisition Multiple Peak-Interval Procedure
}

Nathaniel Rice

West Virginia University

Follow this and additional works at: https://researchrepository.wvu.edu/etd

\section{Recommended Citation}

Rice, Nathaniel, "Adaptation of Temporal Control to Unpredictable Mid-Session Changes in a RapidAcquisition Multiple Peak-Interval Procedure" (2013). Graduate Theses, Dissertations, and Problem Reports. 4995.

https://researchrepository.wvu.edu/etd/4995

This Thesis is protected by copyright and/or related rights. It has been brought to you by the The Research Repository @ WVU with permission from the rights-holder(s). You are free to use this Thesis in any way that is permitted by the copyright and related rights legislation that applies to your use. For other uses you must obtain permission from the rights-holder(s) directly, unless additional rights are indicated by a Creative Commons license in the record and/ or on the work itself. This Thesis has been accepted for inclusion in WVU Graduate Theses, Dissertations, and Problem Reports collection by an authorized administrator of The Research Repository @ WVU. For more information, please contact researchrepository@mail.wvu.edu. 
Adaptation of Temporal Control to Unpredictable Mid-Session Changes in a Rapid-Acquisition Multiple Peak-Interval Procedure

\author{
Nathaniel Rice
}

Thesis submitted to the Eberly College of Arts and Sciences at West Virginia University in partial fulfillment of the requirements for the degree of

Master of Science

in

Psychology

Elizabeth G. E. Kyonka, Ph.D., Chair

Karen G. Anderson, Ph.D.

Julie Patrick, Ph.D.

Morgantown, West Virginia

2013

Keywords: temporal control; timing; peak interval; rapid acquisition; acquisition 


\begin{abstract}
Adaptation of Temporal Control to Unpredictable Mid-Session Changes in a Rapid-Acquisition Multiple Peak-Interval Procedure
\end{abstract}

\title{
Nathaniel Rice
}

The temporal arrangement of events is important in laboratory and real-world settings. Many real-world scenarios are highly dynamic and not reflected by the steady-state nature of experiments often conducted in laboratory settings. To address the dynamic nature of the environment, four pigeons responded on a rapid-acquisition multiple peak-interval schedule. Peak-interval trials, extinction trials that are extended and deliver no reinforcement, were interspersed with fixed-interval trials in a two-component multiple schedule. The interval for each component changed mid-session every session. There were two conditions: an Unsignaled condition where the intervals were changed mid-session without any accompanying stimulus change and a Signaled condition where the mid-session change was preceded by a 5-min blackout. Temporal control, measured by start and stop times from peak-interval trials, developed within each session. The 5-min blackout facilitated acquisition of temporal control by decreasing the influence of the previous interval. Temporal control was reacquired each session half. Discriminative signals facilitated adaptation in a dynamic environment. 


\section{Acknowledgements}

I would like to thank Elizabeth Kyonka, Karen Anderson, and Julie Patrick for serving as members of my thesis committee and for their valuable input and comments in preparing this manuscript. I am especially thankful to Liz for serving as the chair of my committee and as my advisor. 
Table of Contents

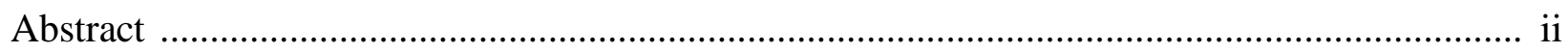

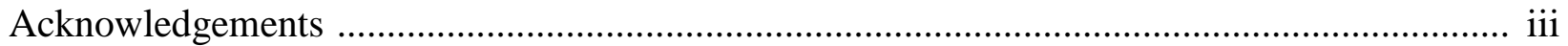

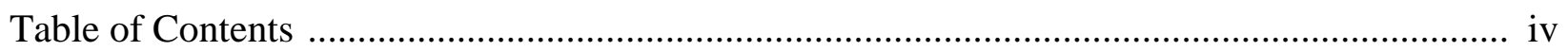

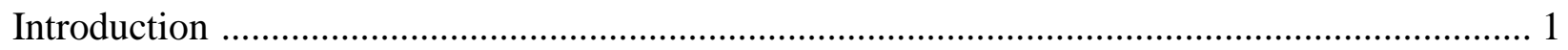

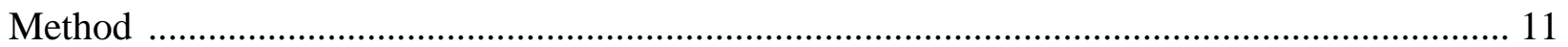

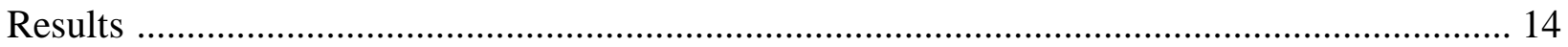

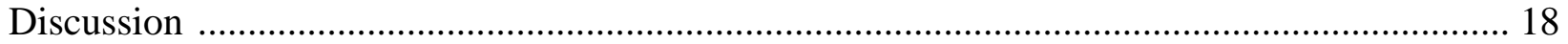

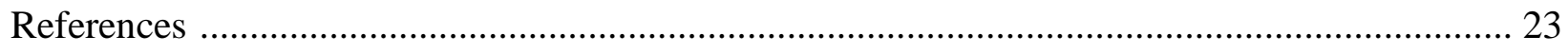

Tables and Figures 


\section{Introduction}

The study of timing is important because all events occur in time. In the laboratory, behavioral scientists arrange reinforcers to have some temporal relation to responses. The timing of everyday behavior is similarly significant. For example, children learn best when reinforcement or punishment immediately follows the behavior that is being modified. Pets learn when food is available by listening for the sound of a can opener or the rustling of a food bag, indicating that the temporal contiguity between these two events is important. By better understanding how behavior changes as time to an event changes, we can better predict and understand behavior in transition. Most behavior in the natural environment is in some transition state - unchanging contingencies rarely govern behavior over long periods of time. So while steady-state responding is noteworthy and important, the study of transitional states of behavior is a significant element of a complete understanding of behavior. The purpose of the current experiment was to study timing in a dynamic environment to better understand transitional behavior.

\section{Fixed-Interval Schedules}

One way of investigating interval timing experimentally is to expose subjects to fixedinterval (FI) schedules of reinforcement. In FI schedules, the first response after an interval has elapsed is reinforced. Steady-state experiments present the same interval over many sessions, where this interval produces stable responding - that is, the response pattern does not change systematically from session to session. Typical steady-state responding in FI schedules is characterized by pausing or responding at a low and constant rate for approximately two-thirds of the interval (Schneider, 1969). When response rates are aggregated over many FI sessions and plotted as a function of time since trial onset, the resulting function is positively accelerated. 
In this function, response rates are highest at the time of reinforcer delivery (Dews, 1978). Analysis of individual trials, however, shows a different pattern of responding. Typically, low and constant rates of responding occur at trial onset. At some point before reinforcer delivery, responding abruptly switches to a high rate and continues until a reinforcer is delivered.

Schneider (1969) labeled this pattern of responding in individual trials a "break-run" pattern.

\section{Peak-Interval Schedules}

When using FI schedules to study interval timing, it is only possible to obtain measures of temporal control from trial onset until reinforcer delivery. For example, consider the time of reinforcer availability in a fixed-interval trial as time $T$, in seconds. As a trial elapses, the time to reinforcement (time to $T$ ) becomes progressively smaller. During this transition, response rates typically increase and reach a maximum at $T \mathrm{~s}$. In a fixed-interval trial, a reinforcer is delivered following the first response at least $T \mathrm{~s}$ after the interval began. Then the trial ends and there is no further opportunity to respond. One limitation of this procedure is that it is impossible to determine what happens after $T$, or what would happen as time since $T$ increases. The peakinterval (PI) procedure (Roberts, 1981) uses modified FI schedules to investigate responding after $T \mathrm{~s}$ has elapsed in a trial. In the PI procedure, there are two types of trials: FI trials and PI trials. FI trials are the same as interreinforcer intervals from a normal FI schedule; a reinforcer is delivered following the first response that occurs after a fixed amount of time has elapsed. In PI trials, reinforcers are never delivered. PI trials are much longer (typically 2-4 times longer; e.g., Cheng \& Westwood, 1993) than FI trials. Responses can occur after the time when a reinforcer would have been delivered (time $T$ ) on an FI trial. The inclusion of these PI trials allows for measurement of timing from trial onset to when a reinforcer would have been delivered (making it equivalent to an FI trial) as well as measuring patterns of responding from the time of 
reinforcement availability to when the subject stops responding or the lengthened trial ends (that is not available in an FI trial). The additional measures of timing make the PI procedure an effective means of determining what variables affect responding and acquisition of responding when temporal factors are of interest.

When PI-trial response rates throughout a trial are aggregated over many sessions, the resulting shape of the response distribution approximates a Gaussian. The distribution has a maximum, or peak, near the programmed time of reinforcer availability on FI trials (Cheng \& Westwood, 1993; Roberts, 1981). In individual PI trials, as in FI trials, responding starts at some low and constant rate. Responding switches to a high rate before reinforcement would have occurred in an FI trial (time $T$ ). The time since trial onset when this switch occurs is called the “start time." Sometime after the reinforcer would have been delivered (time $T$ ), responding once again switches back to some low and constant rate, referred to as the "stop time" (Church, Meck, \& Gibbon, 1994). The response gradients from FI trials were characterized as having break-run patterns. Similarly, gradients from PI trials have break-run-break patterns. The additional switching back to a low rate of responding is available only during PI trials. Figure 1 shows an example response gradient for a single PI trial, with the breaks and run labeled. Start and stop times are denoted by the dashed vertical lines. In this example, time $T$ occurs at $30 \mathrm{~s}$ and is marked by an arrow.

\section{Timing Properties and Timing Acquisition}

Two important scalar properties of interval timing have been demonstrated empirically: timescale invariance and scalar variability. Timescale invariance is a property where measurements of timing are proportional to the mean of the programmed interval. That is, longer intervals will produce relatively longer measures of timing (such as start/stop times, 
pausing, or later inflection points in the Gaussian distribution) than shorter intervals (Lejeune \& Wearden, 2006). Variability, measured as the standard deviation of the response gradient, is directly proportional to schedule value (Church \& Gibbon, 1982; Gibbon \& Church, 1981). In other words, relative variability is constant; this is scalar variability. One way of assessing whether variability is scalar is by calculating the coefficient of variation $(\mathrm{CV})$, which is the standard deviation of the sample divided by its mean. The CVs should remain constant, despite any change in the programmed interval. Zeiler (1985) performed a systematic manipulation of interval durations and found that for intervals between $5 \mathrm{~s}$ and $80 \mathrm{~s}, \mathrm{CV}$ s did not systematically vary.

Much of the empirical research on interval timing has been conducted using steady-state procedures, where the same set of contingencies are used over extended periods of time. In these procedures, data are analyzed when behavior is stable and is no longer in transition. However, in non-laboratory settings behavior is often in transition, limiting the generality of this approach. More recently, efforts have been made to analyze the acquisition of temporal control. Machado and Cevik (1998) analyzed how response rates changed in two groups of pigeons exposed to either an FI 40-s schedule or an FI 80-s schedule. Response rate measures from the beginning of the experiment showed no positive acceleration when aggregated, indicating that temporal control failed to develop. That is, high-rate responding started early in a trial and continued until reinforcer delivery. After increased exposure to the schedules, temporal control began to develop, shown by decreased response rates early in a trial and increased response rates late in a trial, closer to reinforcer delivery. Higa, Wynne, and Staddon (1991) arranged cyclical interfood intervals (IFIs), where the time between reinforcer deliveries was varied according to a sinusoidal function. This experiment involved response-initiated delay schedules, where a 
reinforcer was delivered after some delay, which was initiated by a single peck. The authors used pause time, the latency from trial onset to the first peck, as the measure of temporal control. When IFIs were varied in a cyclical manner from one interval to the next, pause times continually adjusted to track the upcoming IFI. Pause times were proportional to the IFI of the immediately preceding trial, indicating that in this experiment the pause time was determined by the very recent behavioral history.

Across conditions of an experiment by Gallistel, Mark, King, and Latham (2001), reinforcement rate between two levers was set to 1:4, 1:1, or 4:1. Across the different experimental conditions, reinforcement rate either changed rapidly (within a session) or slowly (between sessions). In these conditions, brain stimulation was delivered to rats according to concurrent variable-interval (VI) schedules. Response allocation adjusted to changes in relative reinforcement rate almost immediately. Prior experience with changing contingencies did not dictate the rate at which behavior changed. Instead, the rate of behavior change was controlled by the frequency of the contingency changes. Taken together, these results show that by arranging for rapidly changing contingencies, behavior can be kept in acquisition for extended analyses.

\section{Rapid-Acquisition Procedure}

The label "rapid acquisition" is applied to procedures where an independent variable is changed pseudorandomly every session. For example, in an experiment designed to assess interdependence of choice and timing (Kyonka \& Grace, 2007), terminal-link FI intervals in a concurrent-chains procedure were changed every day. To assess adjustment of choice and timing to the new intervals, response allocation and start and stop times were recorded six times per session. Rapid-acquisition concurrent schedules (Hunter \& Davison, 1985; Schofield \& 
Davison, 1997) and concurrent chains (e.g., Grace, Bragason, \& McLean, 2003) have also been used to investigate relative rate of reinforcement and relative terminal-link immediacy. Rapid acquisition has also been applied to the study acquisition of temporal control in the PI procedure (Rice, Kyonka \& Grace, in prep).

Grace, Bragason, and McLean (2003) used rapid acquisition in a concurrent-chains procedure to investigate the dynamics of choice in pigeons. In their procedure, two white side keys signaled the initial links. Each trial began with a VI schedule, which could be satisfied by a response on one of the two side keys, which was pseudorandomly determined. When a peck satisfied the initial-link schedule, the other side key was darkened. The terminal link was then illuminated on the selected key and the FI terminal link began. As in FI schedules, when the programmed terminal-link interval elapsed, the next peck to the illuminated key produced food. In Grace and colleagues' experiment, terminal-link FI duration was manipulated and new intervals were arranged at the start of each session.

In a series of experiments, Kyonka and Grace (2007, 2009, 2010) obtained convergent measures of acquisition of preference (measured by allocation of responses in the initial links) and temporal control by embedding the PI procedure in terminal links of rapid-acquisition concurrent-chains schedules. Twelve times each session, they calculated start and stop times, which are single-trial measures of temporal control obtained from PI trials. The start time indicated where responding switched from a low rate to high rate and the stop time indicated where responding switched from the high rate back to a low rate.

In the earliest of these experiments, Kyonka and Grace (2007) manipulated the duration of the terminal links while holding the initial-link VI schedule constant. The initial link associated with the shorter terminal link varied pseudorandomly across sessions. In the 
"Minimal-variation" condition, the terminal-link durations were always $10 \mathrm{~s}$ and $20 \mathrm{~s}$. In the "Maximal-variation" condition, the terminal-link durations could vary between $6 \mathrm{~s}$ and $24 \mathrm{~s}$ with the constraint that they always summed to $30 \mathrm{~s}$ each session. Across sessions, start and stop times obtained from PI trials were controlled by the interval duration of the terminal links: longer intervals produced later start and stop times than shorter intervals. The pattern of responding that emerged from the PI trials in both conditions was within-session acquisition of timing. At the beginning of sessions, start times were early (near $0 \mathrm{~s}$ after terminal-link onset) and stop times were late (much longer than the longest possible interval duration). Over the course of a session, start and stop times rapidly adjusted to values that more closely approximated the programmed interval and stabilized by the first half of the session. By the end of the session, start times were later and stop times were earlier in a trial, indicating better temporal control because the values better approximated the interval in effect for that session. There were no differences in acquisition of temporal control between the Minimal- and Maximal-variation conditions.

Subsequent experiments using a similar procedure that varied the relative duration of the initial (Kyonka \& Grace, 2009) and terminal links (Kyonka \& Grace, 2010) showed very similar patterns of choice and timing. The authors regressed start and stop times onto the programmed interval from the current session (Lag 0) and two previous sessions (Lags 1 and 2). Start and stop times were controlled by the current ( $\operatorname{Lag} 0)$ interval. That is, longer intervals were associated with later start and stop times obtained from PI trials. There was no systematic control of start or stop times by interval durations from the previous two sessions (Lags 1 and 2). Thus, the authors posited that subjects approached sessions de novo, rapidly adjusting to the contingencies in place for a session with no savings from previous sessions. 
In another experiment, naïve pigeons were exposed to multiple PI schedules in an attempt to determine whether context played a role in the acquisition of response rates and patterns (Rice, Kyonka, \& Grace, in prep). The purpose of that experiment was to investigate timing without choice, so the initial links were removed and only terminal links were used, effectively presenting a multiple schedule. Here, red and green keys signaled separate PI schedules. Each session was comprised of 72 trials broken into 6 blocks of 12 trials each. Within each block, five FI trials and one PI trial were arranged for each component (red and green). Start and stop times were obtained from PI trials. There were two conditions, "Correlated" and "Uncorrelated." In the Correlated condition, the two intervals summed to 30 s, replicating Kyonka and Grace's (2007) maximal-variation condition. The Correlated condition was so called because the two intervals presented each session were perfectly negatively correlated $(r=-1.0)$. In the Uncorrelated condition, the intervals for the red and green components averaged to $30 \mathrm{~s}$ across all sessions but were not restricted to sum to $30 \mathrm{~s}$ in any given session. The perfect correlation between intervals in the Correlated condition did not facilitate temporal learning: the rate of acquisition and the precision of start and stop times (how well they approximated the interval) were comparable in the Correlated and Uncorrelated conditions.

The behavior pattern that emerges in rapid-acquisition procedures can be used to determine which variables facilitate the acquisition of temporal control. The analyses of timing in Kyonka and Grace (2007) and Rice, Kyonka and Grace (in prep) included calculation of coefficients of variation to assess the degree of scalar variability, multiple regressions to quantify control by current and previous sessions, and measures of precision and sensitivity to illustrate how timing adapted to new intervals within session. These experiments provide a foundation for the present research, in that they demonstrate that start and stop times can adapt to unpredictably 
changing intervals within a session. The present experiment is designed to assess within- and between-session adaptation to new intervals when changes are introduced mid-session.

\section{Statement of the Problem}

Previous rapid-acquisition experiments have investigated temporal control using concurrent-chain schedules (Kyonka \& Grace, 2007, 2009, 2010) or multiple PI schedules (Rice, Kyonka \& Grace, in prep). In previous studies, the intervals between trial or terminal-link onset and reinforcer availability changed unpredictably across sessions but did not change within sessions. Under these conditions, start and stop times from the first half of sessions were relatively conservative. That is, at the beginning of sessions when subjects had limited experience with the intervals, PI trials were characterized by early start times and late stop times. By the end of a session, start times occurred later for relatively long intervals and stop times occurred earlier for relatively short intervals. Thus, start and stop times became more precise in that they came to more closely approximate the programmed interval on FI trials over the course of a session. Additionally, precision of the start and stop times stabilized in the first half of the session.

While previous studies using rapid acquisition have shown that responding on PI trials can adjust to unpredictable changes in interval durations and stabilize within-session, none of these studies have investigated how temporal control changes when intervals are changed midsession instead of at the start of each session. A discriminative stimulus is a stimulus that signals some aspect of potential reinforcement or punishment. Should the start of a session facilitate acquisition of an interval, possibly by acting as a discriminative stimulus, then different effects should be seen in the acquisition of temporal control using this procedure when compared to previous research. Specifically, there should be differences in acquisition of temporal control 
between a condition that signals an interval change (Signaled condition) and a condition that does not (Unsignaled condition).

The major difference between these two conditions is the discriminability of the interval change that occurs mid-session. In the Signaled condition, a 5-min blackout, which should be discriminable, precedes the interval change. In the Unsignaled condition, there is no accompanying stimulus change, so response patterns must change as a function of experience only. If there is a difference in temporal control between these two conditions, it should be most evident immediately after the point in the session when the 5-min blackout occurs in the Signaled condition. At that point, effects of the presence or absence of the blackout will be most prominent. The amount of control exerted by the current interval or the amount of control exerted by the interval that was in effect for the first half of the session, or both, might differ. Specifically, in the Signaled condition, the start and stop times may be controlled by the current interval only because the change was signaled. In the Unsignaled condition, start and stop times may be controlled by both the current interval and the previous interval, as some amount of exposure to the new interval is required before behavior can change. Therefore, the prediction was that stop times in the Unsignaled condition would be controlled partially by the previous and current programmed interval (since the pigeon can only continue to respond to the old contingencies to eventually change to the new) while the stop times from the Signaled condition would be controlled by the current contingencies only, since the blackout served as a signal for separate the two sets of contingencies.

The current experiment used a rapid-acquisition PI procedure to characterize effects of within-session transitions on acquisition of temporal control. The primary objective of the experiment was to determine how within-session and between-session acquisition of temporal 
control changes as a function of when new intervals were introduced and how those changes are signaled. If successful, this research should show how different signals can facilitate acquisition of temporal control by examining the role of the blackout as a discriminative stimulus.

\section{Method}

\section{Subjects}

Four White Carneau pigeons (Columba livia) of mixed sex, numbered 201-204, were maintained at $85 \%$ ad libitum weight plus or minus $15 \mathrm{~g}$ through appropriate post-session feedings and housed individually in cages with a 12-hr:12-hr light:dark cycle with free access to water and intermittent access to grit.

\section{Apparatus}

Four operant-conditioning chambers $(25.5 \mathrm{~cm}$ deep x $32 \mathrm{~cm}$ wide $\times 33.5 \mathrm{~cm}$ high) were enclosed in sound-attenuating boxes containing ventilation fans that provided air circulation and white noise. Each chamber contained three keys $24 \mathrm{~cm}$ above the floor arranged in a row $6 \mathrm{~cm}$ apart, a houselight located on the back wall at the top of the chamber, and a grain magazine with a $5.5 \mathrm{~cm}$ x $6 \mathrm{~cm}$ aperture that was centered $5.5 \mathrm{~cm}$ above the floor. The houselight provided general illumination at all times, except during reinforcer delivery, during all phases of the procedure. The magazine, which was illuminated during reinforcement, contained grain until March, 2011 and Nutriblend pigeon chow thereafter. A force of approximately $0.15 \mathrm{~N}$ was necessary to operate each key. Experimental events were controlled through a computer and MED-PC® interface located in an adjacent room.

\section{Procedure}

Because all subjects had previous exposure to rapid-acquisition procedures, the current experiment began immediately with the multiple PI schedule. With few exceptions, sessions 
were conducted at approximately the same time every day. Sessions ended after 72 trials or 70 min, whichever came first.

The reinforcer was changed from mixed grain to Nutriblend pigeon chow early in the experiment at the request of the veterinary staff. The transition was monitored and no systematic change in start or stop times was observed. The data from the transition were not included in the final analysis, meaning the food reinforcer in all included sessions was Nutriblend pigeon chow.

At the start of each trial, the center key was lighted either red or green, determined pseudorandomly. On FI trials, pecks to the center key were reinforced with 3-s access to food upon satisfaction of the FI schedule. On PI trials, the center key was lighted red or green for 60 s, no matter what the duration of the FI trial was. This ensured that the duration of PI trials could not serve as temporal cues. There was no programmed consequence of a center key peck at any point during these PI trials. After a trial ended, the key light was extinguished. The next trial began after a variable-time 8-s intertrial interval elapsed.

The 72 trials that comprised each session were arranged in blocks of 12 with the constraint that in each block, five FI trials and one PI trial were assigned to each component (red and green). The programmed interval determined the time from stimulus onset to reinforcer availability in FI trials and was different for red and green components each session. Intervals for both components were changed for trial 37 (halfway through a session) and carried over into the next session, effectively presenting an interval for the last half of a session and the beginning half of a session for two consecutive sessions. Figure 2 shows the experimental arrangement, where new intervals are introduced in Block 4 and continue into the following session.

Interval durations in FI trials for each session were determined by the same approach Kyonka and Grace (2007) used to produce terminal-link intervals in their maximal-variation 
condition: pairs of intervals were generated using a pseudorandom number generator subject to the constraints that the intervals ranged from 6 to $24 \mathrm{~s}$ and intervals from the two components summed to $30 \mathrm{~s}$ for each session. Intervals were programmed to sum to $30 \mathrm{~s}$ each session in order to keep rate of reinforcement consistent across sessions. To ensure that average programmed intervals were equivalent across components, the shorter interval in each session was assigned to the red or green component according to the 31-step pseudorandom binary sequence (PRBS) similar to that used by Hunter and Davison (1985).

All subjects were exposed to the Unsignaled condition first, then the Signaled condition. In the Unsignaled condition, intervals were changed mid-session without an accompanying stimulus change. That is, once trial 36 was completed, new intervals were in effect for both components with no programmed stimulus change. In the Signaled condition, the mid-session interval change was preceded by a 5-min blackout, where all keys and houselight were darkened. This signal was implemented to make the transition between intervals more discriminable. All subjects experienced the Unsignaled condition for 103 sessions and the Signaled condition for 93 sessions.

\section{Data Analysis}

All analyses were conducted on data from the final 62 sessions of each condition. Measures of temporal control on individual PI trials were obtained using the method of Cheng and Westwood (1993). Responses from individual PI trials were sorted into 1-s bins. The start time was the time of the first response in the first instance of three consecutive 'filled' bins that each contained at least one response. The time of the last response before three consecutive bins without any responses was designated the stop time. 
These start and stop times were then regressed onto the intervals from FI trials in the current session (the session that the start and stop times came from) to determine the sensitivity of timing to the changing contingencies. Higher unstandardized regression coefficients indicate that the programmed intervals from FI trials had a larger influence on start and stop times. Lower values indicate that start and stop times were not as influenced by the programmed interval. To determine what possible sources of control could account for lower levels of sensitivity (unstandardized regression coefficients) and to determine to what degree start and stop times were controlled by previous sessions, stop times were regressed onto the current interval (Lag 0), the previous interval (Lag 1), and two intervals prior (Lag 2).

If the 5-min blackout facilitated the acquisition of timing, there would be evidence of greater temporal control, a higher level of sensitivity, or possibly more immediate increases in sensitivity in the Signaled condition than in the Unsignaled condition. In addition, multiple regression analyses would show decreased control by previous intervals in the second half of a session when new intervals are arranged, due to the increased discriminability of the transition.

\section{Results}

\section{Temporal Control}

Temporal control developed in all subjects: start and stop times in PI trials changed depending on the programmed interval in FI trials. That is, start and stop times were later in PI trials of schedules with longer FI trials. This is evident in Figure 3, where start and stop times taken from PI trials in each session half are plotted as a function of the programmed interval on FI trials. For all subjects, the slopes for start (range: 0.09 - 0.46) and stop times (range: 0.34 1.07) were non-zero and positive, which indicates that start and stop times changed as a function of the interval in effect on FI trials. In addition, start times changed less than stop times when 
they adjusted to time to reinforcement on FI trials, which is consistent with previous research (Kyonka \& Grace, 2007). Slopes for start and stop times increased as a function of exposure (across blocks) and stop times had systematically higher slopes than start times.

To investigate the acquisition of temporal control, start and stop times from PI trials were regressed onto the programmed interval from FI trials to obtain sensitivity coefficients for each subject, shown in Table 1. These coefficients quantify how much start and stop times differed as a function of the programmed interval. The larger the coefficient, the greater the influence of programmed interval on start or stop time. If stop times were more sensitive to programmed interval when changes in programmed interval were more discriminable, sensitivity coefficients for the Signaled condition would be higher than in the Unsignaled condition. However, sensitivity coefficients were not statistically significantly different in the Unsignaled (start: $M=.21, S D=.08$; stop: $M=.56, S D=.16$ ) and Signaled conditions (start: $M=.26, S D=.09$; stop: $M=.67, S D=.24)$ for either start times, $t(6)=-1.03, \mathrm{p}=.343$, or stop times, $t(6)=-.839, \mathrm{p}=.434$. In addition, there were no systematic differences between sensitivity coefficients for Blocks 1-3 (start: $M=.25, S D=.09$; stop: $M=.66, S D=.17$ ) and Blocks 4-6 (start: $M=.22, S D=.09$; stop: $M=.57$, $S D=.23), t(15)=1.629, \mathrm{p}=.124$. Across subjects, sensitivity coefficients for the first block of exposure to each interval pair (Block 4) were $M=.17(S D=.10)$ for start times and $M=.32$ $(S D=.25)$ for stop times. Sensitivity coefficients from the first block of the session (Block 1) (start: $M=.18, S D=.08$; stop: $M=.40, S D=.13$ ) were comparable to sensitivity coefficients from the first block of exposure, $t(15)=.909, \mathrm{p}=.378$. Although subjects had been exposed to the interval in Blocks 4-6 of the previous session, there was no carryover into Blocks 1-3 the next day. 
Figure 4 shows sensitivity coefficients for start and stop times for each subject and condition. Here, the increases from one block to the next represent acquisition of temporal control. For all subjects, start time coefficients were lower than stop time coefficients. Sensitivity typically increased across session halves as exposure to the interval increased. However, there were instances (stop time sensitivity from Pigeons 201 and 202 in the Signaled condition and Pigeon 204 in both conditions) where the function was bitonic, indicating a different pattern of within-session change to start and stop times than would be expected if acquisition occurred at a steady rate throughout the session. In Figure 4, it is also apparent that acquisition occurred anew each session half, as the functions began at near-zero levels then increased over the session half.

While there were no systematic differences in sensitivity coefficients as a function of block or condition, if there were any differences between the conditions, it would be in most detectable Block 4. This difference would be greater control of stop times by the previous interval (which was in effect for the first half of the session) in the Unsignaled condition. For quantitative characterizations of the control of programmed intervals on stop time, a multiple regression with stop time from PI trials as the outcome variable was computed for each subject from each block of each condition. Predictors included were the interval that was in effect when a stop time was recorded $(\operatorname{Lag} 0)$, the interval that was previously in effect $(\operatorname{Lag} 1)$, and the interval in effect before that ( $\operatorname{Lag} 2)$. The equation for this multiple regression is listed below:

$$
\text { StopTime }=a_{0} F I_{0}+a_{1} F I_{1}+a_{2} F I_{2}+b \quad \text { Equation } 1 .
$$

In Equation 1, the subscripts represent the lag, the three $a$ parameters are the unstandardized regression coefficients, the $b$ parameter is a y-intercept, and $F I$ is the interval taken from FI trials. In previous rapid acquisition research, the current contingencies, or the Lag 
0 interval, exerted the most control over responding and the influence of higher-lag schedules was not statistically significantly different from zero (Kyonka \& Grace, 2007; Rice, Kyonka \& Grace, in prep).

If start and stop times were determined primarily by the FI duration in effect at the time, sensitivity coefficients should be largest for Lag 0 intervals. Lag 2 intervals were temporally distant and therefore should not significantly contribute to current responding. The Lag 1 interval, however, was expected to control start and stop times in the Unsignaled condition because no visual stimulus change was associated with the change in intervals. Instead, experience was the only indicator of the new contingencies. In the Signaled condition, the 5-min blackout was expected to decrease the control by the Lag 1 interval, because it was a signal that the previous interval was no longer in effect.

Figure 5 shows sensitivity coefficients obtained from fitting Equation 1. As predicted, the Lag 1 sensitivity coefficients (gray squares) from Block 4 were consistently higher in the Unsignaled condition (range: $0.31-0.52$ ) than in the Signaled condition (range: $-0.09-0.20$ ), $t(6)=4.12, \mathrm{p}=.006$. Additionally, sensitivity to the Lag 2 interval was consistently near-zero for all subjects; no Lag 2 coefficients $(M=.04, S D=.17)$ were statistically significantly different from zero $(\mathrm{p}=.26-.90)$. The blackout in the Signaled condition made the mid-session interval change more discriminable than it was in the Unsignaled condition: control of start and stop times by the Lag 1 interval decreased. To further illustrate this difference, Figure 6 shows the sensitivity coefficients for stop times in Block 4 for each condition and subject. In each case, the sensitivity coefficient for the Unsignaled condition was higher than the Signaled condition, demonstrating that the 5-min blackout increased the discriminability of the mid-session interval change and decreased control by the Lag 1 interval. 


\section{Discussion}

In this experiment, pigeons were exposed to a multiple PI schedule, where the majority of trials were FI trials that ended in reinforcement. One out of every six trials was a PI trial, where reinforcement was withheld and the subjects could continue to respond until the trial timed out after $60 \mathrm{~s}$. Start and stop times were obtained from these PI trials. The programmed interval in FI trials changed mid-session (after 36 trials) every session. The change was not marked with any accompanying stimulus change in the Unsignaled condition, but in the Signaled condition the change was marked with a 5-min blackout.

Regressions of the start and stop times onto the programmed interval from FI trials showed that temporal control developed within session for all four subjects. Longer FI intervals produced later start and stop times in subsequent PI trials. Additionally, start and stop times adjusted rapidly and stabilized within a session. This acquisition of temporal control was similar to previous work (Kyonka \& Grace, 2007; Rice, Kyonka \& Grace, in prep). After three PRBS sequences, stop time sensitivity coefficients of approximately 1 have been reported in concurrent-chain experiments (Kyonka \& Grace, 2007). By contrast, stop time coefficients in this experiment tended to be lower (range: 0.34-1.07). A potential explanation for this discrepancy was that the terminal-link intervals in Kyonka and Grace's (2007) experiment were more discriminable than those in the present experiment.

Comparisons of the slopes and sensitivity coefficients of start and stop times showed no systematic differences between conditions or sessions halves (Blocks 1-3 vs. Blocks 4-6). However, it was predicted that Block 4 would be the most likely point in the session a difference between conditions would exist because the programmed difference between the two conditions, the blackout, occurred immediately before Block 4. The 5-min blackout in the Signaled 
condition was expected to increase the discriminability of the mid-session change in intervals, thereby allowing faster and more accurate acquisition of temporal control.

To test the hypothesis that the greatest differences in sensitivity coefficients would be observed in Block 4, stop times taken from each block were regressed onto the current programmed interval (Lag 0), the previous interval (Lag 1), and two intervals prior (Lag 2) for each subject. In these regressions, Block 4 sensitivity to the Lag 0 interval was equivalent across conditions. However, Block 4 sensitivity to the Lag 1 interval was lower in the Signaled than the Unsignaled condition. The Lag 1 interval was the interval that was in effect at the beginning of the session. The blackout was a discriminative signal that marked the change to intervals in both components and changed stop times in Block 4. This difference produced no change in control by the Lag 0 contingencies, the programmed interval currently in effect, and less control by the previous, Lag 1 interval. This reduction in control by the Lag 1 interval is adaptive, as responding to the current contingencies of reinforcement are more adaptive than responding being partially controlled by contingencies no longer in effect. In the Signaled condition, stop times were reacquired when a new interval was in effect, whereas in the Unsignaled condition stop times were a function of the current and past contingencies.

Previous rapid acquisition work suggests that measures of timing (e.g., start and stop time) and choice are not influenced by the previous contingencies. That is, the current behavior is a function of the contingencies currently in effect and almost nothing else. In the previous work, schedules of reinforcement were changed at the beginning of a session. An objective of the current experiment was to determine how the timing and signaling of interval changes contribute to this reacquisition of intervals. Specifically, could a 5-min blackout replicate effects of the beginning of a session for acquisition of temporal control, or are the 22 hours of 
nonexperimental time a necessary component of that rapid acquisition? In this experiment, stop times from the Unsignaled condition were influenced by the previous intervals, which is not found in previous work. In the Signaled condition, the blackout decreased control of the previous intervals and produced a pattern of behavior that is similar to previous work. Taken together, this suggests that the blackout functioned similarly as the beginning of a session does for previous rapid acquisition work. Additionally, this signal facilitated acquisition in Block 4.

The current experiment failed to find carryover of sensitivity from the end of one session to the beginning of the next. Figure 4, which shows unstandardized Lag 0 sensitivity coefficients for each subject, condition and block, illustrates the similarity in the functions between the session halves. For both the Unsignaled and Signaled conditions, both session halves show reacquisition of control by the interval in effect. That is, sensitivity increased from some low or near-zero value to a higher sensitivity coefficient over the course of 36 trials (half of a session) for most subjects. If there had been carryover between sessions, stop times from the first half of sessions should have higher sensitivity to Lag 0 intervals than stop times from the second half of sessions. Since the contingencies in effect for the first half of a session were carried over from the previous session, higher sensitivities or more accurate start and stop times could be expected as a result. However, neither of these were found.

Carryover between sessions has occurred in other experiments. In steady-state procedures, the responding adapts to the contingencies and shows a pattern of acquisition over several sessions. In a rapid acquisition experiment, Christensen and Grace (2009) used concurrent chains where one terminal link was always $8 \mathrm{~s}$ and a second terminal link that varied from $2 \mathrm{~s}$ to $32 \mathrm{~s}$. This varying terminal link duration was changed in an ascending or descending sequence. Response allocation in the initial links tracked changes in the terminal links. 
Additionally, they found a hysteresis effect, where the responding was biased toward the 8-s alternative in the descending sequence (which started at $32 \mathrm{~s}$ ) and biased towards the varied alternative in the ascending sequence (which started at $2 \mathrm{~s}$ ).

While Christensen and Grace (2009) arranged for a dynamic environment, their terminallink values changed according to a non-random sequence. In their experiment, response allocation was biased according to the immediately preceding intervals on the varying terminal link, perhaps as a function of this non-random sequence. A short terminal link produced a bias towards that key even when the varying alternative became longer and the leaner schedule. The opposite is also true - a long terminal-link interval produced a bias towards the 8-s terminal link, even when the varying alternative decreased in programmed interval duration and became the richer schedule. So, even in a dynamic environment, carryover of response patterns between sessions is possible. However, a key feature might be that the environment in Christensen and Grace's (2009) experiment changed predictably, while the environment in the current experiment changed pseudorandomly.

It is possible that the mid-session interval changes produced a pattern of responding most fit for a dynamic environment - reacquisition. In Kyonka and Grace’s (2007) earlier work, when the terminal link intervals were $10 \mathrm{~s}$ and $20 \mathrm{~s}$, pigeons reacquired the intervals every session. In the current experiment's replication of their Maximal-variation condition, where terminal links varied from $6 \mathrm{~s}$ to $24 \mathrm{~s}$, there was also reacquisition. This pattern of responding is expected given the previous research, but the current experiment introduced these changes mid-session. Because the intervals in effect were the exact same as the previous session, it would be adaptive to continue responding in the same way as the previous session. It is adaptive in the sense that responding could better reflect the contingencies and the pattern or number of responses could be 
diminished, thereby saving effort. However, that did not occur here, as evidenced by new acquisition at each session half instead of only when new intervals were in effect. It is unclear whether this failure to carryover between sessions is a function of the nonexperimental time that separated the sessions, the beginning of a session serving as a discriminative stimulus to reacquire the intervals, or some other unconsidered factor.

One possible way to explain why response patterns failed to carry over between sessions is that the complexity of the current task favored new acquisition. In steady-state environments, response patterns persist across sessions, suggesting that the complexity or nature of the current task reinforced responding that adjusted within-session. This is adaptive in an environment that continually changes reinforcement contingencies, as this experiment did. However, not all aspects of the environment were changed, thereby leading maladaptive responding, as start and stop times had to adjust every new session. It is possible that the discriminability of the sessionto-session carryover was too low for responding to adjust in this manner. Further research should investigate how signals can be implemented to facilitate this carryover and generate more adaptive patterns of responding.

The current experiment implemented the rapid-acquisition procedure in a novel way and has uncovered a seemingly maladaptive, or perhaps less adaptive, pattern of responding. The response patterns failed to carryover between sessions, despite the adaptive nature of doing so. Signaling the mid-session interval change produced more accurate acquisition within Block 4, which demonstrates how acquisition of temporal control was affected by the available discriminative stimuli in this highly dynamic environment. In non-laboratory settings, which are typically dynamic environments, signals can help produce faster acquisition and can potentially lead to more accurate or precise responding. 


\section{References}

Cheng, K., \& Westwood, R. (1993). Analysis of single trials in pigeons' timing performance. Journal of Experimental Psychology: Animal Behavior Processes, 19(1), 56-67.

Church, R. M., \& Gibbon, J. (1982). Temporal generalization. Journal of Experimental Psychology: Animal Behavior Processes, 8(2), 165-186.

Church, R. M., Meck, W. H., \& Gibbon, J. (1994). Application of scalar timing theory to individual trials. Journal of Experimental Psychology: Animal Behavior Processes, 20(2), $135-155$.

Dews, P. B. (1978). Studies on responding under fixed-interval schedules of reinforcement: II. The scalloped pattern of the cumulative record. Journal of the Experimental Analysis of Behavior, 29(1), 67-75.

Gallistel, C. R., Mark, T. A., King, A., \& Latham, P. E. (2001). The rat approximates an ideal detector of changes in rates of reward: Implications for the law of effect. Journal of Experimental Psychology: Animal Behavior Processes, 27(4), 354-372.

Gibbon, J., \& Church, R. M. (1981). Time left: Linear versus logarithmic subjective time. Journal of Experimental Psychology: Animal Behavior Processes, 7(2), 87-108.

Grace, R. C., Bragason, O., \& McLean, A. P. (2003). Rapid acquisition of preference in concurrent chains. Journal of the Experimental Analysis of Behavior, 80(2), 235-252.

Higa, J. J., Wynne, C. D., \& Staddon, J. E. (1991). Dynamics of time discrimination. Journal of Experimental Psychology: Animal Behavior Processes, 17(3), 281-291.

Hunter, I., \& Davison, M. (1985). Determination of a behavioral transfer function: White-noise analysis of session-to-session response-ratio dynamics on concurrent VI VI schedules. Journal of the Experimental Analysis of Behavior, 43(1), 43-59. 
Kyonka, E. G. E., \& Grace, R. C. (2007). Rapid acquisition of choice and timing in pigeons. Journal of Experimental Psychology: Animal Behavior Processes, 33(4), 392-408.

Kyonka, E. G. E., \& Grace, R. C. (2009). Effects of unpredictable changes in initial-link duration on choice and timing. Behavioural Processes, 81(2), 227-232.

Kyonka, E.G. E., \& Grace, R. C. (2010). Rapid acquisition of choice and timing and the provenance of the terminal-link effect. Journal of the Experimental Analysis of Behavior, 94(2), 209-225.

Lejeune, H., \& Wearden, J. H. (2006). Scalar properties in animal timing: Conformity and violations. The Quarterly Journal of Experimental Psychology, 59(11), 1875-1908.

Machado, A., \& Cevik, M. (1998). Acquisition and extinction under periodic reinforcement. Behavioural Processes, 44(2), 237-262.

Machado, A. (1997). Learning the temporal dynamics of behavior. Psychological Review, 104(2), 241-265.

Rice, N., Kyonka, E. G. E., \& Grace, R. C. (2012). Within-session acquisition in a rapid acquisition multiple peak-interval procedure is equivalent for correlated and uncorrelated schedules. Unpublished manuscript.

Roberts, S. (1981). Isolation of an internal clock. Journal of Experimental Psychology: Animal Behavior Processes, 7(3), 242-268.

Schneider, B. A. (1969). A two-state analysis of fixed-interval responding in the pigeon. Journal of the Experimental Analysis of Behavior, 12(5), 677-687.

Schofield, G., \& Davison, M. (1997). Nonstable concurrent choice in pigeons. Journal of the Experimental Analysis of Behavior, 68(2), 219-232. 
Zeiler, M. D. (1985). Pure timing in temporal differentiation. Journal of the Experimental Analysis of Behavior, 43(2), 183-193. 
Table 1. Mean slopes (sensitivity coefficients). Mean start and stop times based on the three PI trials in the first (Blocks 1-3) or second (Blocks 4-6) half of each session were regressed onto programmed intervals from FI trials. Separate regressions were computed for each dependent variable, subject and condition.

\begin{tabular}{|c|c|c|c|c|c|}
\hline \multirow[b]{2}{*}{ Pigeon } & & \multicolumn{2}{|c|}{ Blocks 1-3 } & \multicolumn{2}{|c|}{ Blocks 4-6 } \\
\hline & & Start & Stop & Start & Stop \\
\hline \multirow{2}{*}{201} & Unsignaled & 0.15 & 0.65 & 0.09 & 0.34 \\
\hline & Signaled & 0.21 & 0.53 & 0.17 & 0.57 \\
\hline \multirow{2}{*}{202} & Unsignaled & 0.29 & 0.79 & 0.35 & 0.69 \\
\hline & Signaled & 0.27 & 0.93 & 0.36 & 1.07 \\
\hline \multirow{2}{*}{203} & Unsignaled & 0.17 & 0.41 & 0.19 & 0.57 \\
\hline & Signaled & 0.42 & 0.57 & 0.20 & 0.38 \\
\hline \multirow{2}{*}{204} & Unsignaled & 0.25 & 0.61 & 0.19 & 0.44 \\
\hline & Signaled & 0.25 & 0.81 & 0.23 & 0.51 \\
\hline
\end{tabular}




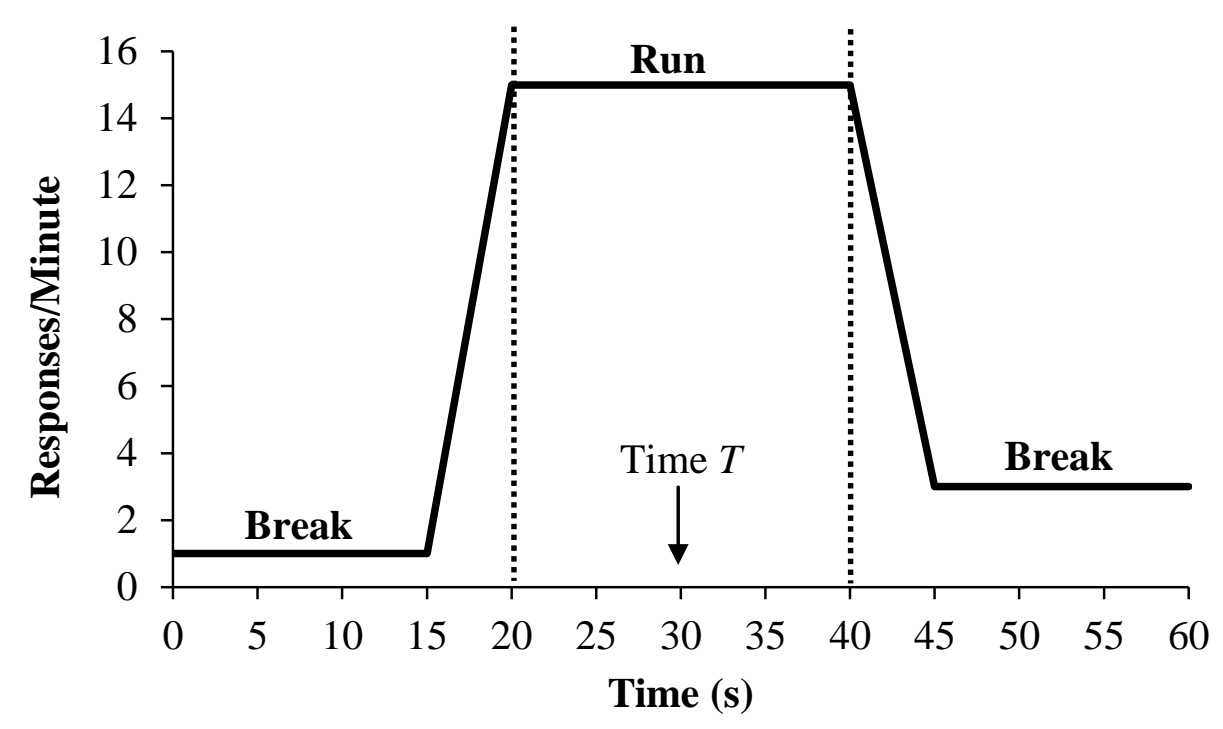

Figure 1. Example response gradient for a single peak-interval trial. Breaks (periods of low responding) and the run (period of high responding) are labeled. Dashed lines indicate the time of transition between two states. The first dashed line marks the start time and the second dashed line marks the stop time. Time $T$, the time of reinforcement availability in a fixed-interval trial, is marked by an arrow and occurs at $30 \mathrm{~s}$ in this example. 


\section{Unsignaled Condition}
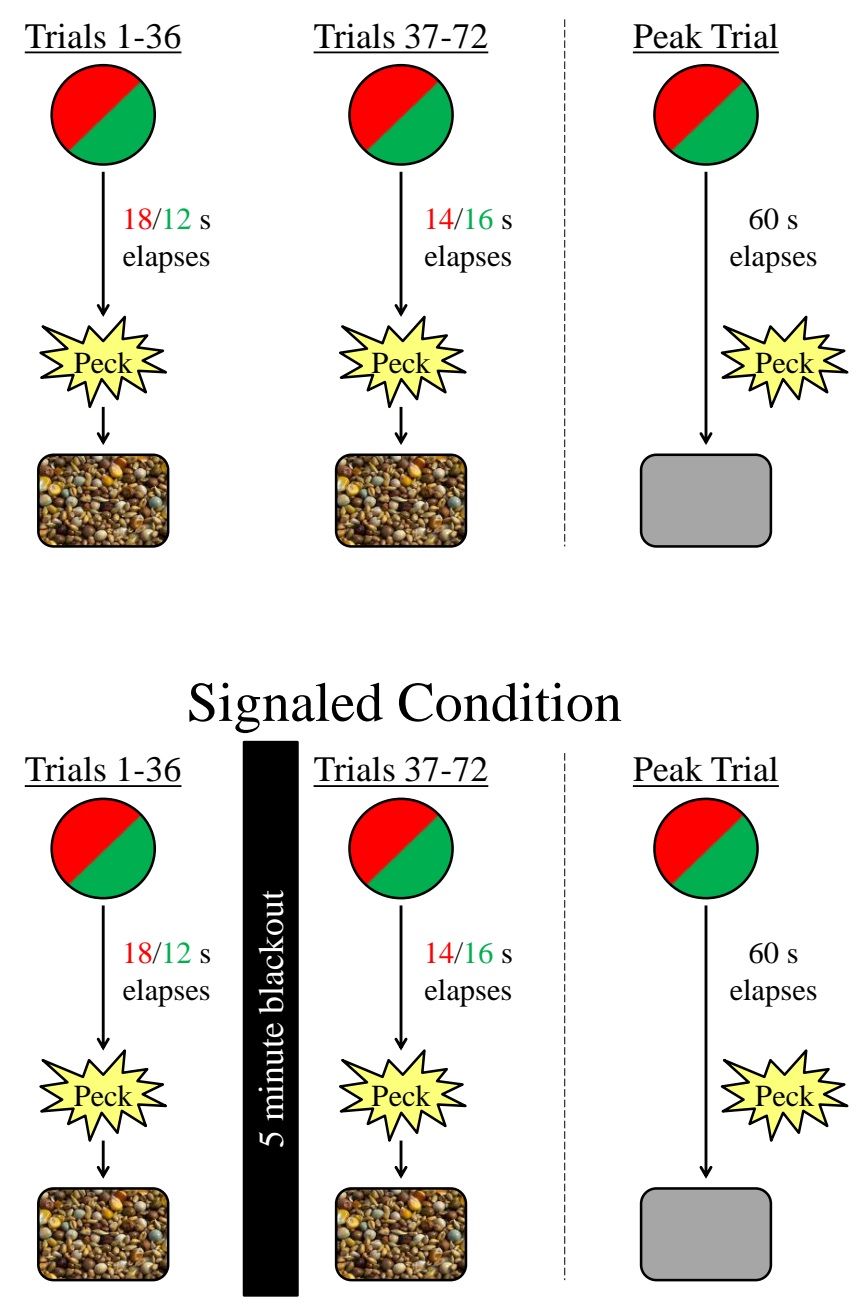

Figure 2. Diagram of experimental procedure for both conditions. Intervals in fixed-interval trials were changed mid-session and carried over into the first half of each new session, effectively presenting an interval for a full session split over two session halves. The Unsignaled condition presented no accompanying stimulus change with the mid-session interval change, while the Signaled condition presented a 5-min blackout that preceded the mid-session interval change. Fixed-interval trials delivered a reinforcer to the first response after the interval had elapsed since trial onset. Peak-interval trials lasted for $60 \mathrm{~s}$ and never ended in reinforcement. 

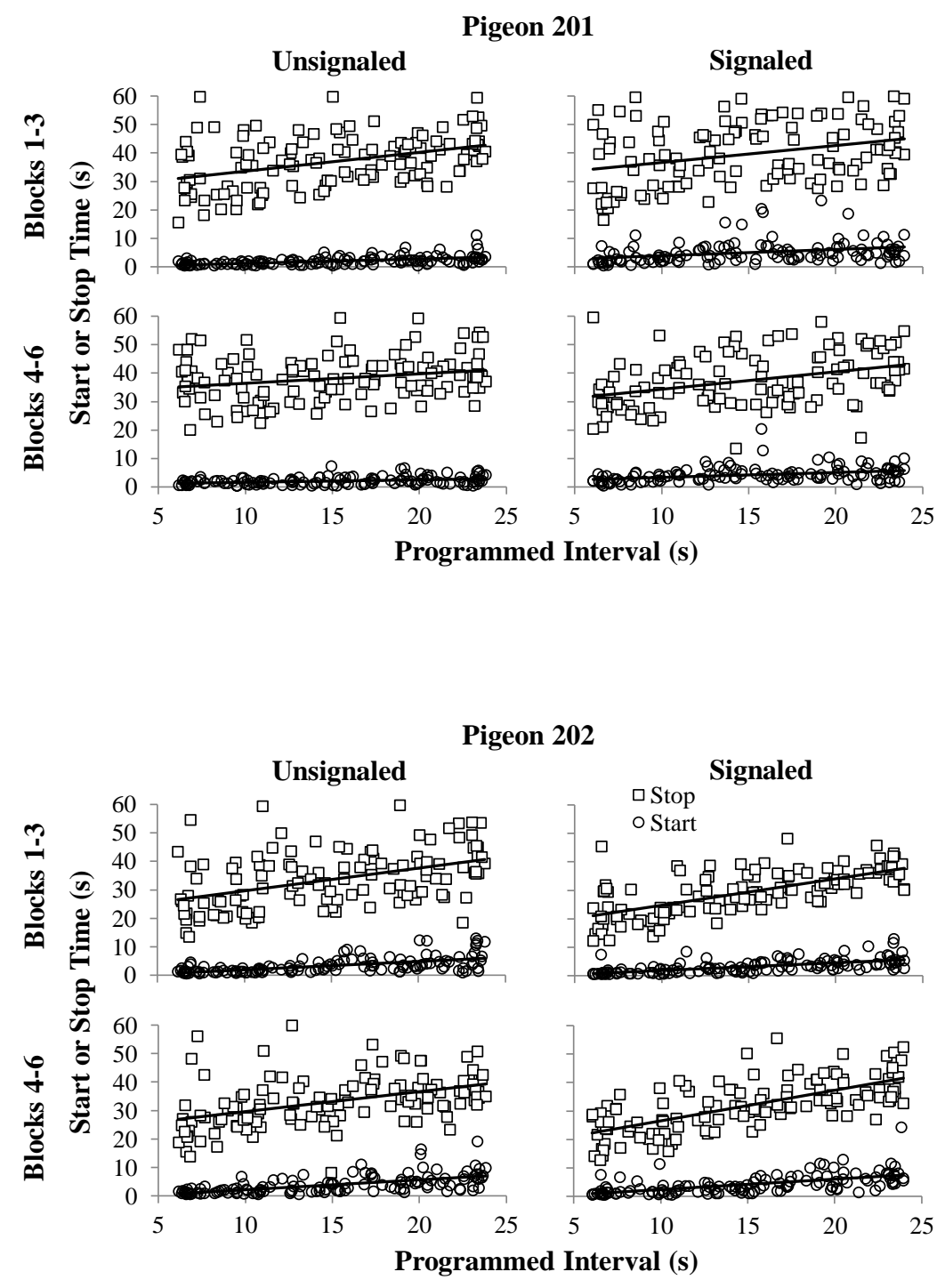

Figure 3. Part 1 of 2 (follows on next page). 

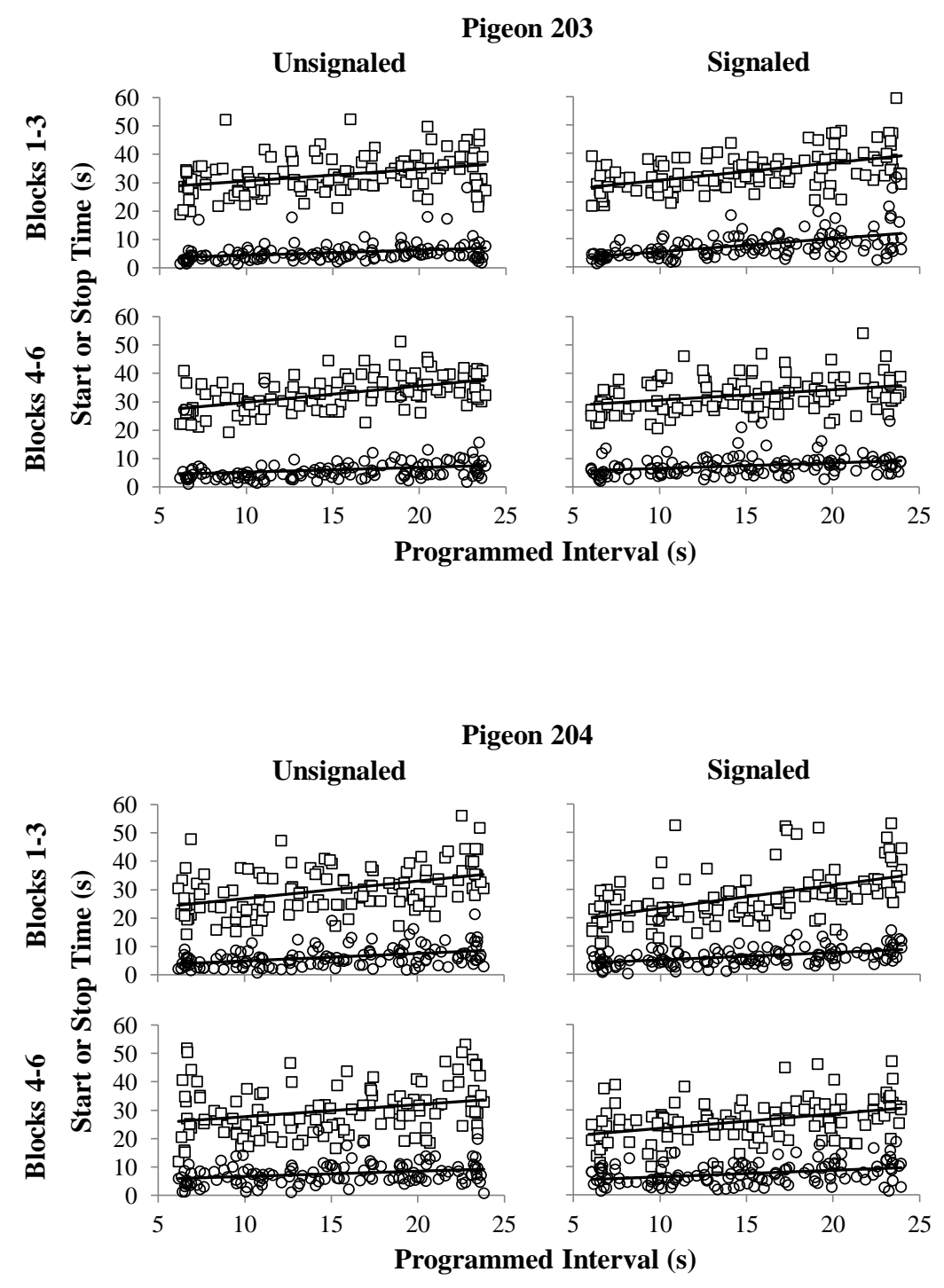

Figure 3. Mean start (filled squares) and stop (empty squares) times taken from peak-interval trials as a function of the programmed interval on fixed-interval trials. Start and stop times were the mean from each session half - Blocks 1-3 are displayed in the top panels and Blocks 4-6 are displayed in the bottom panels. The Unsignaled condition is displayed in the left panels and the Signaled condition is displayed in the right panels. Each data series also displays the best-fitting regression line. 


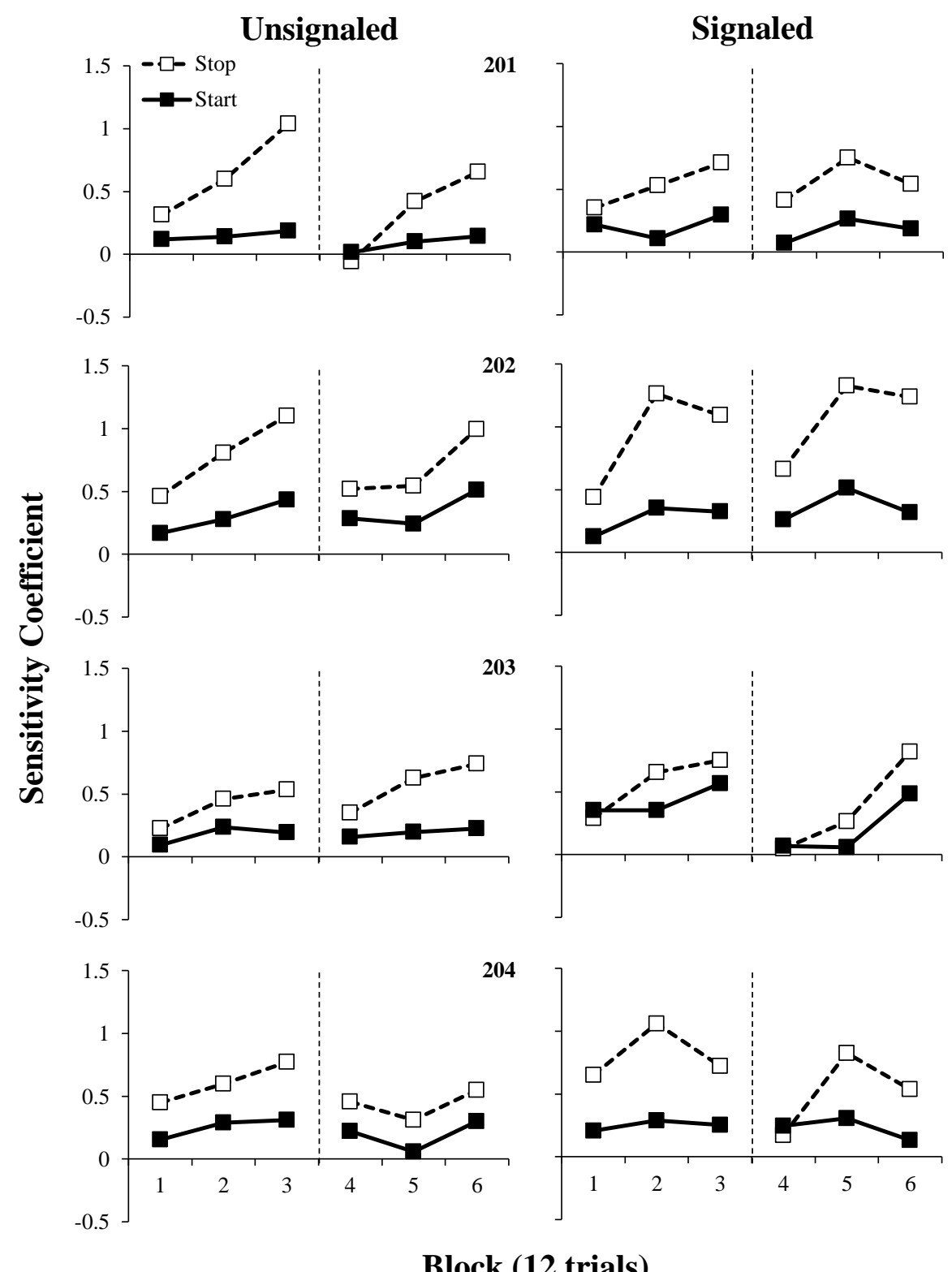

Figure 4. Unstandardized sensitivity coefficients for start (filled squares) and stop (empty squares) as a function of 12 trial blocks for all subjects and conditions. Unstandardized sensitivity coefficients were calculated by regressing the start and stop times from peak-interval trials onto the programmed interval on corresponding fixed-interval trials. 


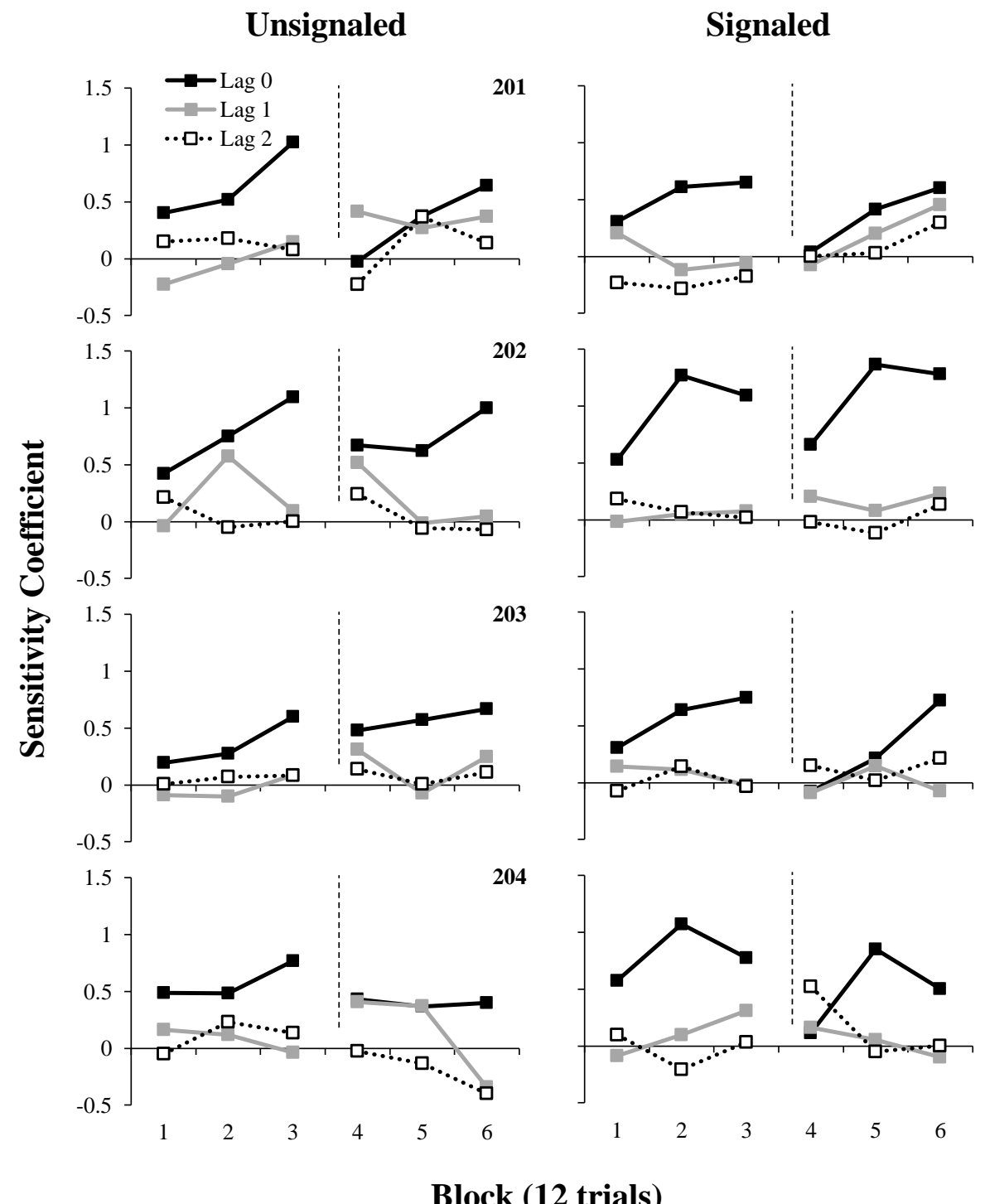

Block (12 trials)

Figure 5. Unstandardized sensitivity coefficients for stop times as a function of block for all subjects and conditions. Additionally, each lag interval (taken from FI trials) is represented by a separate data series. The Lag 0 interval (black squares) is the interval that was in corresponding fixed-interval trials. The Lag 1 interval (gray squares) is the interval that was previously in effect. The Lag 2 interval (empty squares) is the interval that was in effect two schedules prior. 


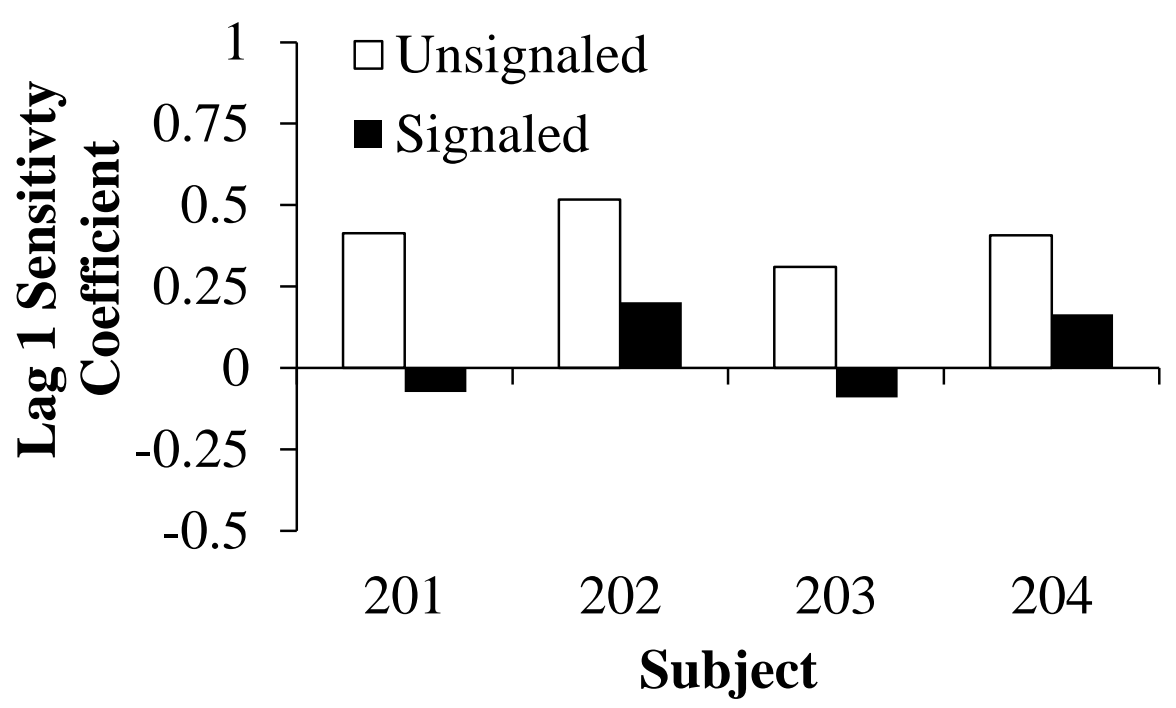

Figure 6. Sensitivity coefficients for stop times from Block 4 regressed onto the Lag 1 interval from FI trials. Empty bars represent the Unsignaled condition and filled bars represent the Signaled condition. 